\title{
A Security Wireless Monitoring and Automatic Protection System for CCEL
}

\author{
Qiao-yan Sun, ${ }^{1}$ Xue-jiao Liu, ${ }^{2}$ Yu-mei Sun, ${ }^{1}$ Mei-chun Wang, ${ }^{1}$ Xiang Han $\mathbb{D},{ }^{2}$ \\ and Xiang-guang Chen $\left(^{1,2}\right.$ \\ ${ }^{1}$ Department of Electrical and Electronic Engineering, College of Engineering, Yantai Nanshan University, Longkou 265713, China \\ ${ }^{2}$ School of Chemistry and Chemical Engineering, Beijing Institute of Technology, Beijing 100081, China \\ Correspondence should be addressed to Xiang-guang Chen; xgc1@bit.edu.cn
}

Received 9 December 2020; Revised 15 March 2021; Accepted 1 May 2021; Published 15 May 2021

Academic Editor: Chi-Hua Chen

Copyright (C) 2021 Qiao-yan Sun et al. This is an open access article distributed under the Creative Commons Attribution License, which permits unrestricted use, distribution, and reproduction in any medium, provided the original work is properly cited.

\begin{abstract}
A wireless monitoring and automatic protection system for laboratory safety is proposed to solve the security problems in chemistry and chemical engineering laboratories (CCEL). The system is based on a wireless sensor network (WSN), including indoor wireless security monitoring node, information aggregation and management node, portable handheld terminal, and laboratory security information management system. Each indoor safety wireless monitoring node can monitor the laboratory security information (for example, fire information, toxic gas content, abnormal sound in chemistry, and chemical engineering laboratory) and control the corresponding protection device. The portable handheld terminal can remind the safety responsible person to check the safety status of the laboratory remotely and can issue corresponding operation and control instructions. According to the instructions it sends, the indoor security wireless monitoring and control node can start the corresponding automatic protection device. The experimental results demonstrate that the system can realize all-weather online monitoring of indoor safety information, ensuring that some important scientific research experiments can be conducted continuously and safely, significantly reduced the workload of laboratory managers, and precisely reported the location and conditions of accidents; the loss caused by the accident can be minimized based on the automatic protection device. The system can also be used in dangerous goods warehouse and inflammable and explosive places.
\end{abstract}

\section{Introduction}

At present, the CCELs in many universities and colleges in China are used for both teaching and scientific research, and the number of laboratories for teaching and scientific research is increasing rapidly, and the safety problems of laboratories are worthy of attention [1]. Based on the current status, doing a good job in laboratory safety management, studying the early-warning system of laboratory safety accidents, etc., are all important contents of laboratory safety management in colleges and universities [2]. Effective laboratory safety management measures can ensure the smooth progress of school teaching and scientific research, which is of great significance for students to form correct concepts of the world, life, and values [3].

Reference [4] summarized the laboratory safety education and training, safety management system and guarantee facilities, safety management level, and so on and proposed a number of practical measures to strengthen laboratory safety management. Reference [5] made a brief comparison between China and the United States in the aspects of student safety education, laboratory construction, chemical management, laboratory management, and establishment of management institutions. Reference [6] introduced that some universities in China had achieved good results in the safety management of chemistry and chemical laboratory by referring to the advanced management mode and management system of foreign countries. In order to improve the reliability and practicability of the wireless safety monitoring system, reference [7] had diagnosed the fault of wireless safety monitoring system in chemistry and chemical engineering laboratory.

At present, the management information of the laboratories in some research institutes and universities in China 
cannot be released in time. It is indeed difficult to assess laboratory conditions and environments [8]. Unadvanced monitoring and management system will occupy more resources, and the facilities of the system are limited. The unadvanced databases cannot meet the requirement of most of the experimental facilities and cannot be used for the relevant information management system. In order to ensure the safety of workers and technicians, some students at Chongqing University have designed a system that may not only use sensors to monitor changes in the concentration of gases on site but also automatically select suitable ventilation facilities for different levels of danger to reduce the content of flammable and explosive gases [9, 10]. Reference [11] designed a laboratory remote monitoring device, which achieves the real-time monitoring and gives the automatic voice alarm and alert message to the remote area or host area by using wireless network technology. This work is designed using a PIC16F877 microcontroller and focuses on sensing the existence of the critical parameters and issuing the alarm if those parameters are sensed. Reference [12] proposed a safety information monitoring and data management system, the purpose of which is to monitor the laboratory and obtain relevant data, judge experimental environment data, and transmit relevant sound-light alarm information. The system integrates some intelligent methods in the management of the laboratory and has certain reference value in the safety and telemetry of the laboratory. Reference [13] proposed a real-time detection and automatic alarm system for laboratory status information, which its purpose is to monitor real-time data and laboratory status, automatically determine the relevant environmental indicators, detect whether there is an external intrusion, and configure the sound-light alarm device. The monitoring system can communicate wire line or wirelessly with PC and can realize remote monitoring, which can meet the needs of unattended duty in related laboratories. Reference [14] presented an embedded laboratory security monitoring system. The system uses a camera to obtain video information and a sensor to obtain environment-related information and sends the data to the network server through the wireless sensor network. When an emergency occurs, the alarm information can be displayed on the web, and the sound and light alarm information can be sent. The system can also be used to monitor laboratories or places where valuables are stored. Besides, a wireless remote monitoring system for the laboratory was designed and developed in reference [15].

Laboratories are the very important places for personnel training and scientific and technological innovation in a university. Reference [16] systemically discussed telecommunications, standard management of chemistry laboratory security based on three aspects of logistical support system, management responsibility system, and education security system. The safety operation of CCEL is the key of the CCEL management system. Reference [17] analyzed safety system construction, safety hardware guarantee, and safety protection skills training of CCEL. Reference [18] comprehensively discussed the importance of experimental safety education, whose purpose is to require relevant personnel to improve CCEL safety management ability and ensure the safety of the CCEL environment. Reference [19] indicated to pressingly study laboratory safety management measures, because the prolonged teaching experiment time and the unproperly disposed wastes in the laboratory are all laboratory safety problems.

The authors of reference [20], aiming at the frequent occurrence of laboratory accidents in Chinese universities at present, took chemical engineering laboratory as an example and put forward corresponding safety management methods from four aspects: equipment, reagents and consumables, water and electricity, and rules and regulations. The authors of reference [21] analyzed the main problems existing in chemical engineering laboratories in China, summarized the types of safety accidents in chemical engineering laboratories in Chinese universities in recent years, and put forward a set of effective laboratory safety management mechanism. The authors of reference [22] studied the safety management of chemical engineering laboratories in China; normalized the management of safety education mechanism, safety supervision and evaluation mechanism, prevention and control mechanism, and accident emergency treatment mechanism; and proposed a safety access management system for chemical engineering laboratories. The authors of reference [23] discussed the methods and measures of chemical engineering laboratory safety management, such as the implementation of student safety education, strengthening the construction of chemical engineering laboratory safety rules and regulations, construction and management of safety facilities, and strengthening the management and use of hazardous chemicals, and put forward the hierarchical management of chemical engineering laboratories and the establishment of a "three-level safety inspection" system.

The main references in recent years are the following: the authors analyzed some problems existing in the management of chemistry and chemical engineering laboratory and put forward some improvement schemes [24]: (1) implement scientific management and further improve rules and regulations, (2) establish reward and punishment mechanism to further strengthen safety management, (3) use the Internet to strengthen instrument management and resource utilization, and (4) constantly improve the quality of the laboratory management team.

According to the problems commonly existing in university laboratories in China, the authors put forward several improvement methods [25]: (1) formulate a reasonable and effective safety management system, (2) strengthen the construction of laboratory safety equipment, and (3) strengthen laboratory safety education and training.

The authors pointed out the types of accidents in university chemical engineering laboratories [26]: for example, (1) fire accident, (2) explosion accident, and (3) chemical poisoning accidents. And put forward several improvement measures: (1) strengthen the safety education of students, eliminate people's unsafe behavior, (2) to do a good job in quality management and eliminate the unsafe state of materials, and (3) strengthen the laboratory safety management and conduct the laboratory safety management according to the rules. 
The authors compared Chinese and Norwegian laboratories in five aspects: safety education, safety facilities and management, chemicals management, operation management, and equipment management [27]. It was pointed out that safety education, experimental facilities, drug management, and management should be strengthened and improved.

According to the author's review of relevant statistical data [28], in the past 10 years, there have been 14,539 school fires in China, killing 29 people and injuring 50 others. At the same time, the authors pointed out the problems existing in laboratory safety management in colleges and universities, such as (1) relaxation of safety education process, (2) the dynamic supervision of hazard sources lags behind, and (3) lack of emergency plan. Several improved schemes were proposed, such as (1) introducing online safety training courses, (2) realize dynamic supervision of hazard sources, and (3) virtual simulation of an emergency drill.

In recent years, our research group has completed several research projects in the fields of research and application of WSN and published some articles; the articles [29-34] are representative.

At present, the main safety problems in chemistry and chemical engineering experiments are as follows:

(1) Fire Accident. Since chemistry and chemical engineering laboratories are places for chemical reactions, which are easy to cause fires due to heat release, flammable gas leakage, or aging of electrical equipment, etc. If not timely alarm and take effective firefighting measures, it will cause casualties and property losses

(2) Accident of Chemical Poisoning. In the process of chemistry and chemical engineering experiments, chemicals are needed. There are many toxic substances in these chemicals and they are frequently used. If students lack safety awareness and fail to carry out effective protection in the experiment, they are prone to poisoning. If they fail to report to the police in time and take effective protection measures, death accidents will occur

(3) Explosion Accident. Because there are so many chemicals in a chemical engineering laboratory, the reactions of the chemicals give off heat and produce energy. If the amount of chemical substances used in the experiment is not used properly, it will easily explode. If the alarm is not timely and effective protection measures are not taken, it will cause heavy casualties and property losses

For some chemistry or chemical engineering experiments, it often takes a day or days to complete. During the experiment, if a fire (or poisoning, or explosion) occurs in the laboratory, how can the remote monitoring center be notified in time? How to report the exact location of the accident in time? How can effective measures be taken to protect the accident site? These problems have not been well solved in the chemistry and chemical engineering laboratories of Chinese universities.
In this paper, we proposed a wireless monitoring and automatic protection system for chemistry and chemical engineering laboratory safety. The system is based on a wireless sensor network (WSN), including indoor wireless security monitoring node, information aggregation and management node, portable handheld terminal, and laboratory security information management system. Each indoor safety wireless monitoring node can monitor the laboratory security information (this is fire, poisonous and harmful gas, abnormal explosion sound, and so on) and control the corresponding protection device. When the safety wireless monitoring node detects the unsafe information in the laboratory, it will upload the field information in time and start the indoor safety automatic protection device. The portable handheld terminal can remind the safety responsible person to check the safety status of the laboratory remotely and can issue corresponding operation and control instructions. According to the instructions it sends, the indoor security wireless monitoring and control node can start the corresponding automatic protection device. The laboratory security information management system receives the information transmitted by all wireless monitoring nodes in the laboratories, and processes, analyzes, and stores the data of each wireless monitoring node in the laboratories. The experimental results demonstrate that the system can realize allweather online monitoring of indoor safety information, ensuring that some important scientific research experiments can be conducted continuously and safely, significantly reduced the workload of laboratory managers, and precisely reported the location and conditions of accidents; the loss caused by the accident can be minimized based on the automatic protection device. The system proposed in this paper can also be used in dangerous goods warehouse and inflammable and explosive places in the process of industrial production.

The rest of this paper is structured as follows. Section 2 provides monitoring and control system structure design in CCEL, including a schematic structure of wireless monitoring and protection system, design of indoor security wireless monitoring node, design of portable handheld terminal, and structural design of laboratory safety information management system. Section 3 proposes a security wireless monitoring and automatic protection method in CCEL. Section 4 presents the safety judgment procedures of CCEL. Section 5 discusses the application examples, including the structure of the experimental system, testing of security monitoring node in CCEL, and remote testing based on handheld terminal. Section 6 summarizes the research work of the whole paper and puts forward some constructive proposals for further and in-depth research work in the future.

\section{Monitoring and Control System Structure Design}

Figure 1 shows a schematic structure of a CCEL wireless monitoring and protection system. The system comprises several indoor security wireless monitoring nodes, an information aggregation management node, several portable 


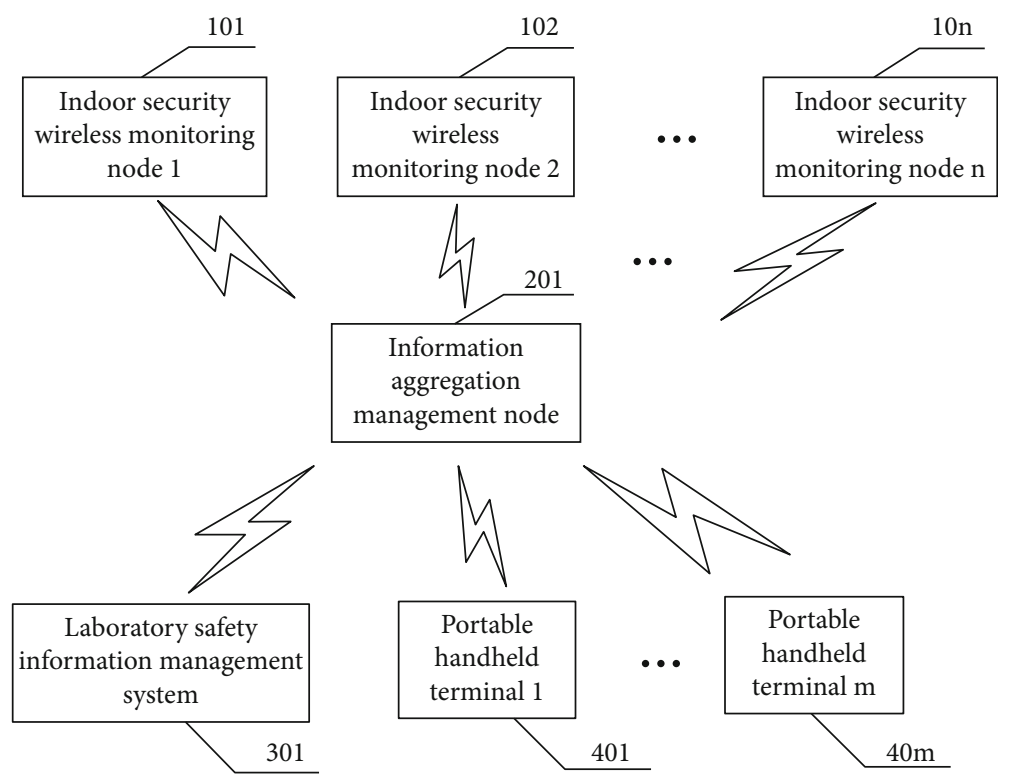

FIGURE 1: A schematic structure of wireless monitoring and protection system in the CCEL.

handheld terminals, and the laboratory safety information management system.

Each indoor security wireless monitoring node in Figure 1 is used to monitor the CCEL security state and to control the automatic protection equipment corresponding to the indoor monitoring node according to the monitoring situation or the instructions of the portable handheld terminal or CCEL information management system. The monitoring CCEL security state includes monitoring the fire information, the poisonous and harmful gas information, and the explosion sound information in the CCEL environment.

The information aggregation management node in Figure 1 is used to support each CCEL security wireless monitoring node to communicate with the corresponding handheld terminal and the laboratory safety information management system. The CCEL safety information management system is used for receiving the monitoring data of the CCEL security wireless monitoring node which is transferred by the information aggregation management node and processing, analyzing, and storing the monitoring data. The handheld terminal in Figure 1 is used for receiving the monitoring data of the CCEL security wireless monitoring node corresponding to the handheld terminal which is transferred by the information aggregation management node and remotely controlling the automatic protection device corresponding to the CCEL security wireless monitoring node.

The CCEL wireless monitoring and protection system may be provided with an indoor security wireless node in each laboratory, such as the indoor security wireless nodes 101 10n in Figure 1, respectively, correspond to $n$ laboratories; then, you can set an information aggregation management node (201) and a laboratory safety information management system (301), the laboratory safety information management system (301) is a subsystem of the laboratory wireless monitoring system, which can be used to record the whole indoor security wireless node data for backup, analysis, and other operations. The system would also pro- vide several portable handheld terminals, that is, $m$ portable handheld terminals 401 to $40 \mathrm{~m}$ in Figure 1 . In this system, $n$ indoor security wireless nodes correspond to $m$ portable handheld terminals, and the corresponding relationship can be one-to-one or many-to-one, which means one portable handheld terminal can manage one CCEL security wireless node, or several portable handheld terminals manage several multiple indoor security wireless nodes.

CCEL security wireless monitoring nodes (101 10n) are applied to monitor the indoor environment temperature, smoke and flame, poisonous and harmful gases, and explosion sound.

The information aggregation management node (201) receives the monitoring information from each laboratory and transmits the monitoring information to the CCEL safety information management system (PC host computer) and the portable handheld terminal, respectively.

The laboratory safety information management system (301) receives the monitoring information from each laboratory transmitted by the information aggregation management node, and processes, analyzes, and saves the information.

The portable handheld terminal $(401 \sim 40 \mathrm{~m})$ receives the monitoring information from each laboratory transmitted by the information convergence management node, monitors the situation in the laboratory through the handheld terminal, communicates with the indoor security wireless monitoring node $(101 \sim 10 \mathrm{n})$, and realizes remote monitoring of the laboratory automatic protection devices.

We can divide the system shown in Figure 1 into four levels. Level 4 is portable handheld terminal. Typically, a laboratory manager corresponds to a terminal or several terminals. That is, he or she is responsible for the safety of a laboratory or several laboratories. Level 3 is a laboratory safety information management system; it is a safety monitoring center for laboratories. It manages all laboratory safety in a building or several buildings. Once a laboratory has an 


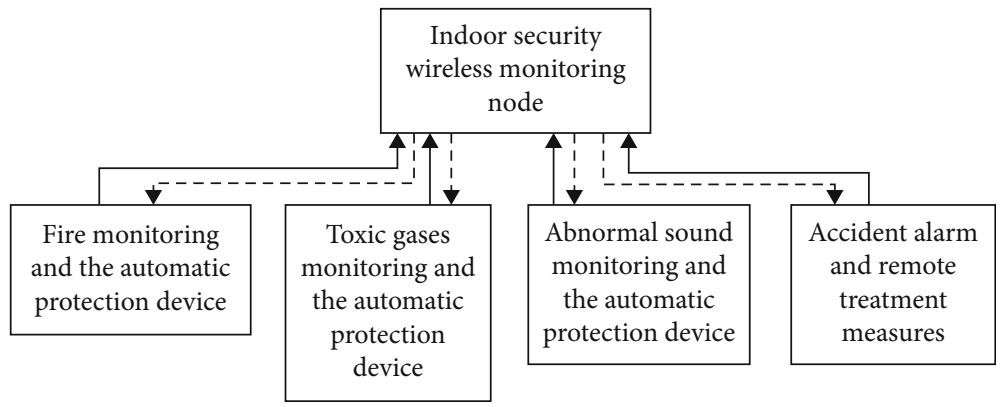

FIgURE 2: Overall functional structure of each indoor security wireless monitoring node.

accident, the monitoring center will contact the person in charge of the handheld terminal in time. They will take the solution as soon as possible.

\subsection{Design of Indoor Security Wireless Monitoring Node.} Figure 2 illustrates the overall functional structure of the indoor monitoring node. Including the indoor security wireless monitoring node control modes: fire, toxic gases, abnormal sound monitoring and the corresponding automatic protection device, and the accident alarm and remote treatment measures, such as sending alarm information to the appropriate portable handheld terminal, receiving remote control commands to activate the automatic protection device.

(1) Sensors. For the detection of temperature, smoke, and flame in the indoor environment, for the detection of toxic and harmful gases in the indoor environment, and for the detection of abnormal sounds in the indoor environment (such as light detonation, explosion, and detonation)

(2) Controller Module. It is used to collect the field data of CCEL, analyze and judge the data, and control the indoor safety automatic protection device and send alarm information

(3) Communication Module. Transmit the parameter information and image information obtained by CCEL on site to the security information management system in CCEL and handheld terminal. Moreover, the command or control information of the handheld terminal and the security information management system in CCEL can be transmitted to the indoor security wireless monitoring node

Each indoor safety wireless monitoring node in CCEL can monitor the laboratory safety status online, can analyze and judge the obtained node monitoring data, and can control the automatic protection equipment through the safety wireless monitoring node. At the same time, the monitoring data will be sent to the security information management system in CCEL, and the corresponding handheld terminal will be sent to remind the security responsible person to conduct remote viewing and determine the corresponding measures, and instructions can be sent to the CCEL wireless monitoring node through the handheld terminal, so as to timely start the corresponding indoor automatic protection device.

In the practical application of this system, each CCEL indoor security wireless monitoring node can transmit the data to the information aggregation management node and, then, send the data from the information aggregation management node to each portable handheld terminal and the security information management system in CCEL. Each security wireless monitoring node in CCEL can also communicate and transmit information with the corresponding portable handheld terminal. When a node fails, it will not affect the normal operation of other nodes, information aggregation management nodes, portable handheld terminals, and laboratory security information management system. Moreover, the information aggregation management node and the portable handheld terminal can be used to determine whether a node has a fault, which can obviously improve the reliability and security of the whole system.

2.2. Design of Portable Handheld Terminal. Figure 3 shows the schematic diagram of the handheld terminal. The handheld terminal includes processor module, communication module, power supply module, liquid crystal display, and keyboard (or touch keyboard or touch screen). It obtains the indoor detection information and image information through the communication module, analyzes the detection information based on the judgment algorithm, determines the type of the accident, and remotely controls the corresponding safety protection device, by looking at the scene images of the accident and timely reporting of the accident site and field conditions.

The solid line shown in Figure 3 indicates the information transmitted to the handheld terminal by the field wireless monitoring node, and the broken line indicates the control information sent out by the handheld terminal to the indoor security wireless supervisory node or the accident type alarm information sent from the handheld terminal to the real field.

2.3. Structural Design of Laboratory Safety Information Management System. The laboratory safety information management system shown in Figure 4 includes a laboratory security wireless monitoring node (301-1), a PC of security information management system (301-2), and a laboratory safety information management system server (301-3). 


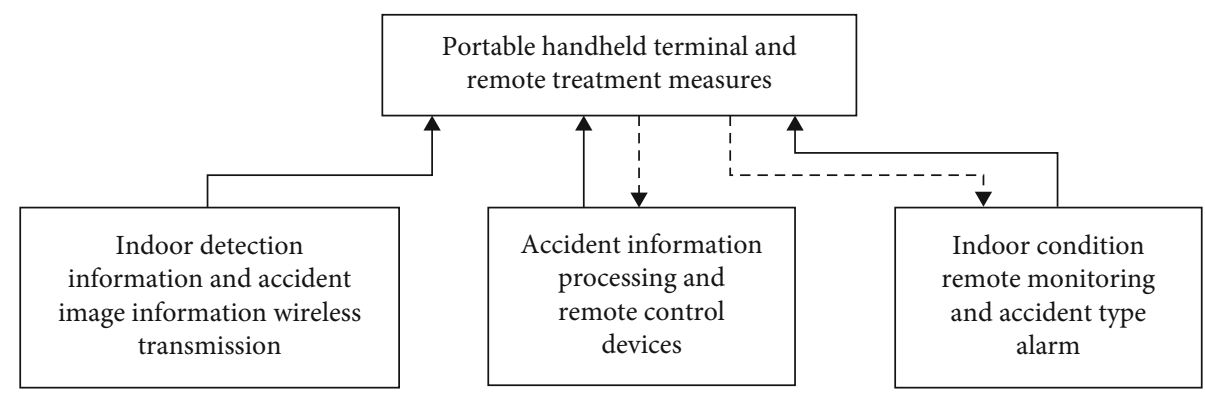

FIGURE 3: The schematic diagram of the portable handheld terminal.

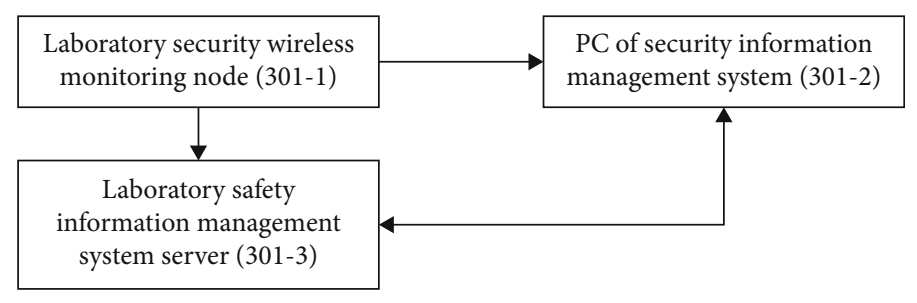

FIgURE 4: Structure of laboratory safety information management system.

The indoor information transmitting and receiving node (301-1) is used for receiving the monitoring data of the indoor monitoring node which is sent by the information aggregation management node and sending the monitoring data to the server (301-3) or the PC host computer (301-2) and sending the instruction of the PC host computer (3012 ) to the information aggregation management node.

The server (301-3) is used for storing the monitoring data received by the indoor information transmitting and receiving node and calls the corresponding stored data according to the instruction of the PC host computer (301-2).

The PC host computer (301-2) is used for providing an exhibition and management interface for displaying the monitoring data and receiving the operation instruction of the manager.

In the system, the indoor information transmitting and receiving node is also called a gateway. It is composed of a processor module, communication module, and node power supply module. It is connected with a PC. Its main function is data receiving and sending.

In the embodiment, a set of management software is provided in the PC host computer for providing the display and the management interface for displaying the monitoring data and receiving the operation instruction of the manager.

The portable handheld terminal cannot store the nodes' data for a long time; in order to analyze the cause of the accident and to back up the remote control, the laboratory safety information management system is designed for backup and analysis of the monitoring data. The information management system receives the detection information from each laboratory transferred from the information aggregation management node, and processes, analyzes, and saves the information.

In addition, in this system, the control modes of automatic protection device also will be designed in the node, and the automatic protection device includes the fire extin- guishing device, the ventilator, and the dangerous goods isolation device.

\section{Security Wireless Monitoring and Automatic Protection Method in CCEL}

This paper proposes a method of wireless monitoring and automatic protection for safety in CCEL. The realization process of this method includes the following steps:

(1) When the fire situation is detected by the safety wireless monitoring node installed in the CCEL room, the fire situation information obtained is analyzed, and the first automatic protection device is determined to be activated according to the analysis results

(2) When the toxic gas information is detected by the safety wireless monitoring node installed in the CCEL room, analyze the toxic and harmful gas information obtained, and determine whether to start the second automatic protection device based on the analysis results

(3) When abnormal sound information is detected by the security wireless monitoring node installed in the CCEL room, the abnormal sound information obtained shall be analyzed, and the third automatic protection device shall be activated according to the analysis results

(4) When the above information is detected by the security wireless monitoring node installed in the CCEL room, the obtained information is transmitted to the security information management system in CCEL through the information aggregation management node and to the handheld terminal corresponding to the indoor security wireless monitoring node 


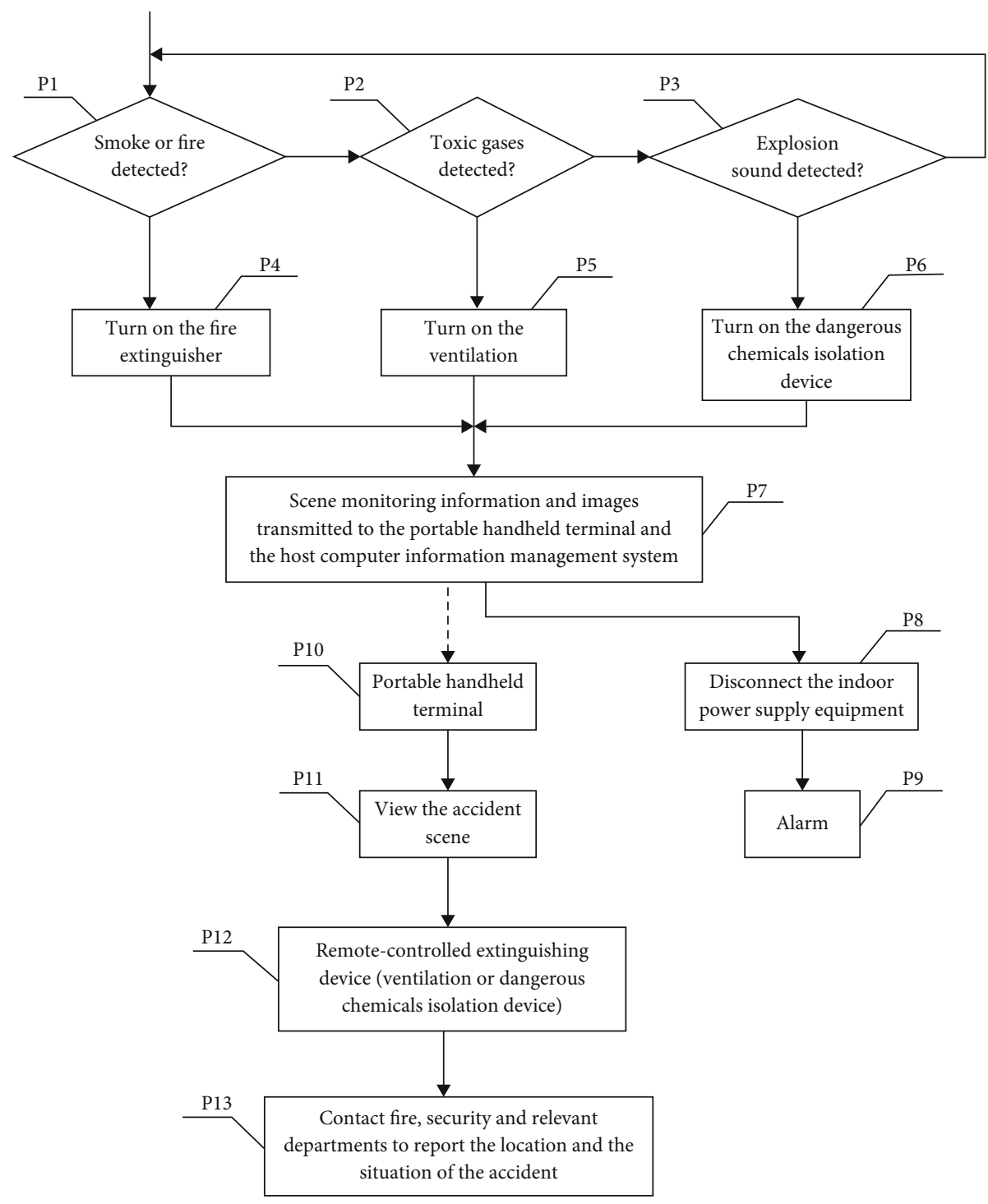

FIGURE 5: Flow chart of laboratory safety wireless monitoring and automatic protection method.

(5) Indoor safety can show by indoor security of wireless data transmission of video monitoring node, the researchers can also use a portable handheld terminal to send the instructions to the indoor security wireless monitoring nodes and can also send functional instructions to the corresponding indoor security wireless monitoring node through information gathering management node. The node controls the relevant automatic protection device and sends the relevant alarm information at the same time

In this system, when a security monitoring node in the CCEL enters the working state, it can simultaneously or successively perform steps (1) to (3), and the order of steps (1) to (3) can be changed, not be restricted.

Figure 5 illustrates the flow chart of a secure wireless monitoring and automatic protection method proposed in this paper. Each step function is described as follows:
P1 Description: determine whether smoke or flame information is detected? If there is, then turn on the fire extinguishing device; if not, then P2 judgment

P2 Description: determine whether toxic gases information is detected? If there is, then turn on the ventilation; if not, then

P3 Description: determine whether abnormal sound information is detected? If there is, then turn on the dangerous chemicals isolation device; if not, then P1 judgment

P4 Description: after confirming by the judgment algorithm, the fire extinguishing device is controlled by indoor security wireless monitoring node, which includes high pressure water and fire extinguishing agent

P5 Description: after confirming by the judgment algorithm, the indoor security wireless monitoring node controls the ventilation device, the ventilation device includes an exhaust fan and a fume hood

P6 Description: after confirming by the judgment algorithm, the dangerous chemicals isolating device is controlled by the indoor security wireless monitoring node, the isolating 


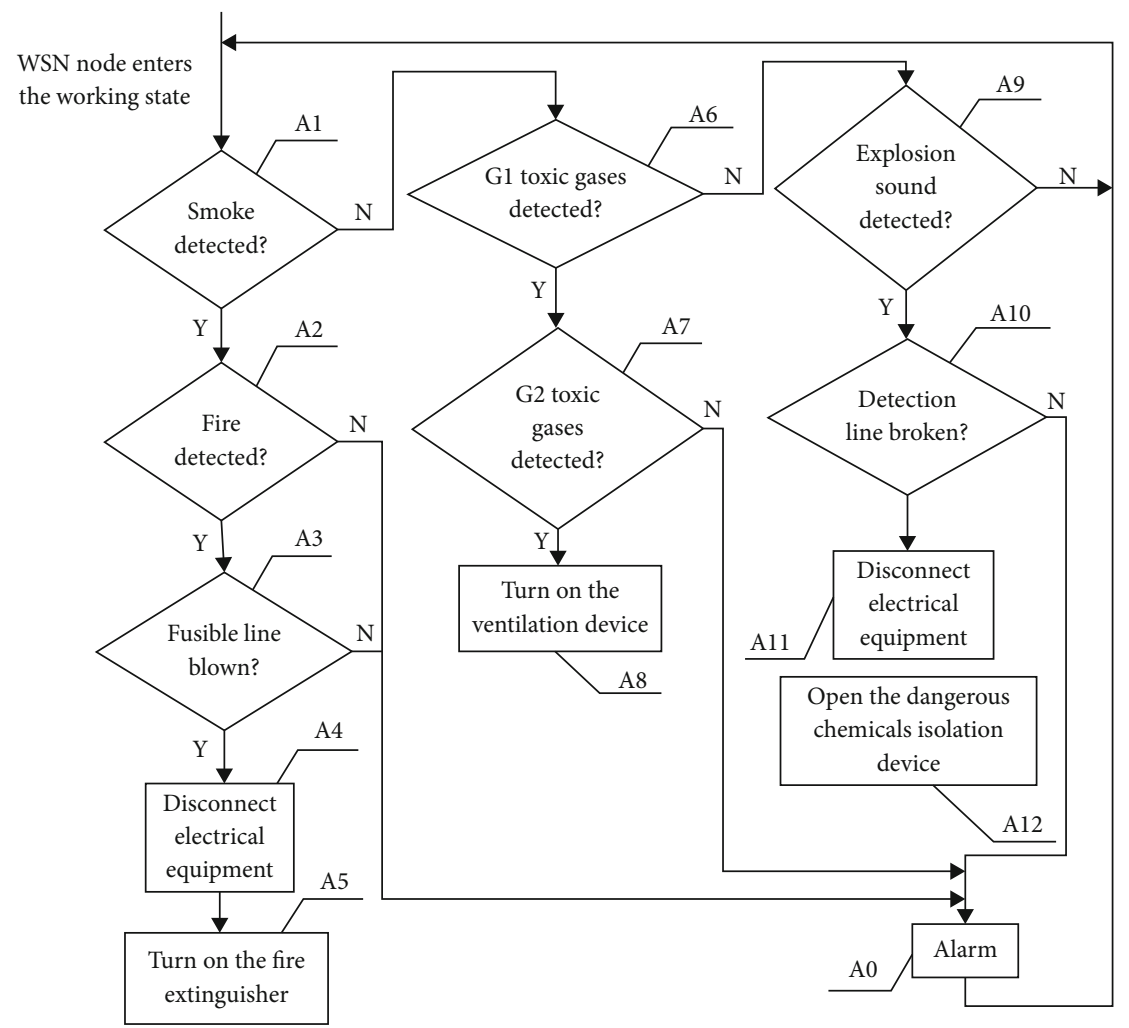

FIgURE 6: The flow chart of the security judgment method in CCEL.

device includes fire-proof material, flameproof material, and explosive-proof material

P7 Description: when the accident occurs, on-site inspection information and images will be transmitted to the handheld terminal and laboratory safety information management system for timely alarm, the effective handling of accidents and analyzing accident cause

P8 Description: disconnect the indoor power supply equipment, to avoid causing other accidents

P9 Description: send out an alarm signal, to facilitate the rapid departure of laboratory staff

P10 Description: quickly view digital and images information when a laboratory alarm is received

P11 Description: the accurate location of the accident can be determined by data and alarm information, and the image information of the accident site can be viewed to determine the remote control scheme

P12 Description: minimize accident losses by remotely operating fire extinguishing devices (ventilation devices or isolating devices)

P13 Description: contact fire department, security department, and other departments in time, and send the exact location of the accident and the relevant information of the accident site

In Figure 5, judgment box P1 judges the temperature, smoke, and flame detected in the indoor environment; judgment box P2 makes judgments on toxic and harmful gases (such as carbon monoxide, nitric oxide, hydrogen sulfide, sulfur dioxide, and chlorine) detected in the indoor environment; judgment box P3 is used to judge abnormal sounds (such as light detonation, explosion, and detonation) detected in the indoor environment.

In Figure 5, (1) through P4 judgment, it is confirmed that when an indoor fire occurs, different fire-extinguishing devices, including high-pressure water, foam fireextinguishing agent, and dry powder fire-extinguishing agent, shall be controlled by indoor safety wireless monitoring node, etc. (2) Judging from P5, when the indoor toxic gas leakage is confirmed, different ventilation devices are controlled by the indoor safety wireless monitoring node, including the exhaust fan, the fume hood, and the automatic opening of Windows. (3) According to P6 judgment, when the indoor explosion sound is confirmed, different dangerous chemical isolation devices shall be controlled by the indoor security wireless monitoring node, including flameretardant materials, flameproof materials, and explosiveproof cover.

\section{Safety Judgment Procedures of CCEL}

The flow chart of the CCEL indoor safety judgment algorithm proposed by us is shown in Figure 6. In order to improve the accuracy of node judgment and the effectiveness of node control, the specific steps and instructions of the algorithm are as follows:

A1 Description: to determine whether smoke information has been detected? If so, enter A2; if not, enter A6 judgment

A2 Description: to determine whether flame information has detected? If so, enter A3 judgment; if not, switch on P0 to alarm and transfer to A1 for judgment 


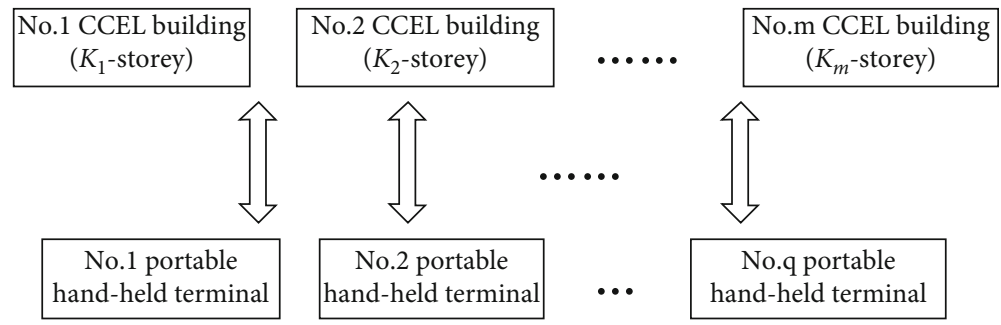

FIGURE 7: The structure chart of handheld terminal monitoring laboratory buildings.

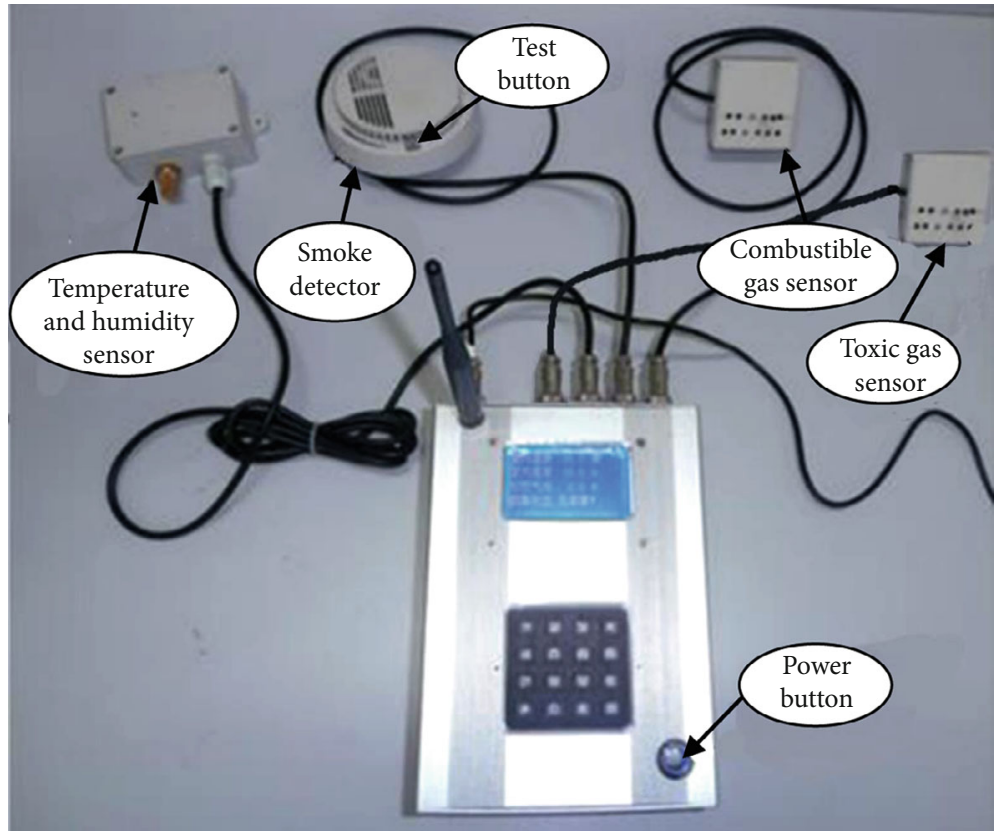

Figure 8: The experimental device of an indoor security monitoring node connected to several sensors.

A3 Description: is it detected that the fusible line has been burnt? If it is burnt out, switch to A4; if it is not burnt out, connect P0 alarm and transfer to A1 for judgment

A4 Description: disconnect the power supply of indoor electrical equipment in the CCEL and switch to A5

A5 Description: activate the fire extinguishing device in the CCEL and transmit the field information and images to the portable handheld terminal

A6 Description: to determine whether G1 has detected toxic and harmful gas? If so, enter A7 judgment; if not, enter A9 judgment

A7 Description: determine whether G2 has detected toxic and harmful gas? If so, enter the A8; if not, A0 alarm is turned on and A1 judgment is transferred

A8 Description: open the ventilation device in the CCEL room and send the field information and images to the portable handheld terminal

A9 Description: to determine whether explosion sound information has been detected? If so, enter the A10; if not, transfer to A1

A10 Description: judge whether the detection line is broken or not? If broken, enter A11; If it is not broken, A0 is connected to alarm and $\mathrm{A} 1$ is transferred to judgment
A11 Description: disconnect the power supply of indoor electrical equipment in the CCEL and switch to A12

A12 Description: activate the isolation device in the CCEL and transmit the field information and image to the portable handheld terminal

AO Description: connect the alarm device of CCEL, namely sound alarm, voice alarm, etc.

The above is a description of each component in Figure 6. Of course, relevant steps for judging fire situation, relevant steps for judging toxic and harmful gas, and relevant steps for judging abnormal sound are at the same level, and the sequence can be changed.

\section{Application Examples}

5.1. Structure of the Experimental System. The schematic diagram of handheld terminal monitoring laboratory buildings is shown in Figure 7, and it allows multiple managers to monitor all laboratories at the same time. Assuming that there are $m k_{i}$-storey $(i=1,2,3 \cdots, m)$ CCEL buildings and $q$ handheld terminals, every terminal can receive all experiment information in $m$ buildings. That means the WSN 


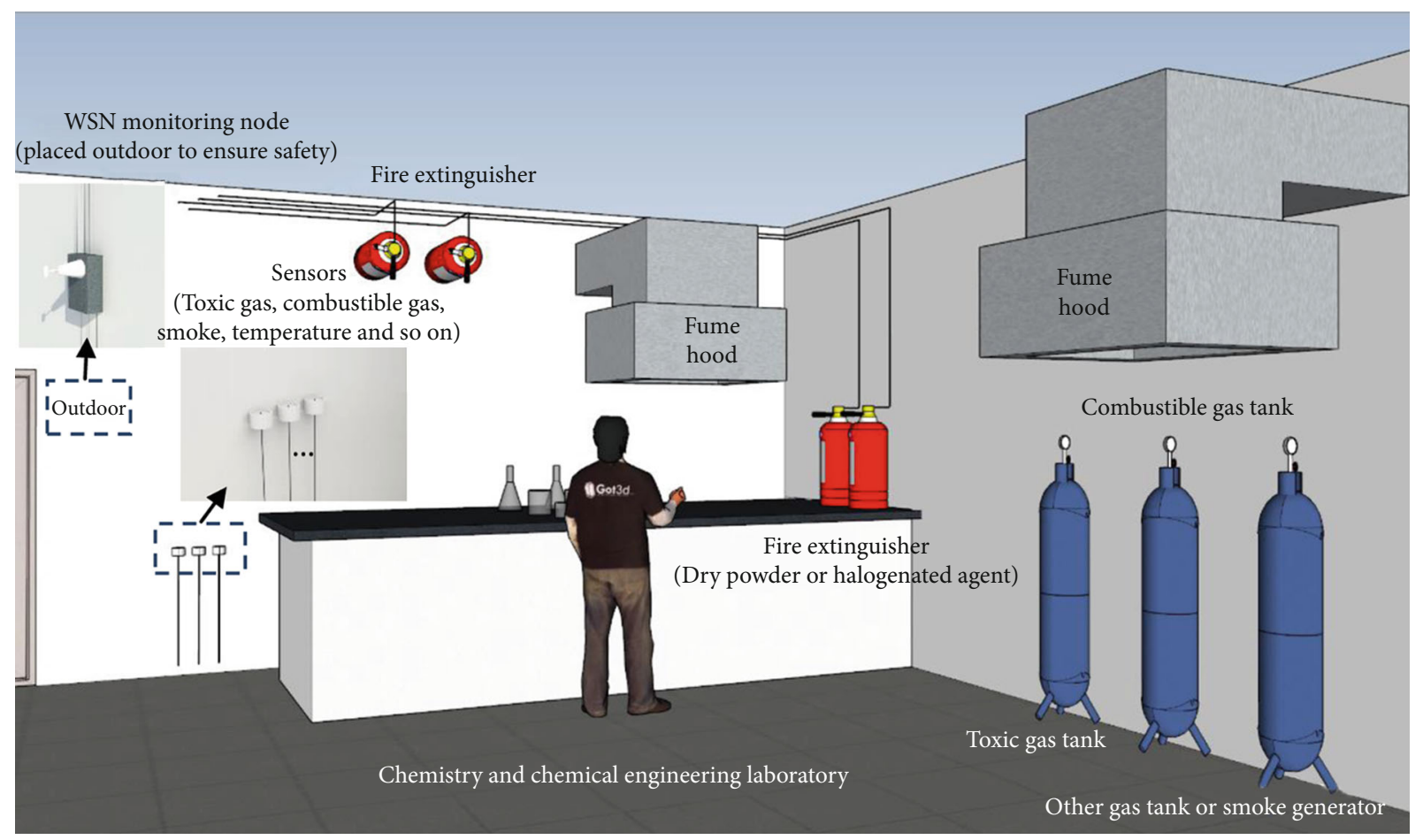

FIGURE 9: The experimental wireless monitoring system diagram for CCEL.

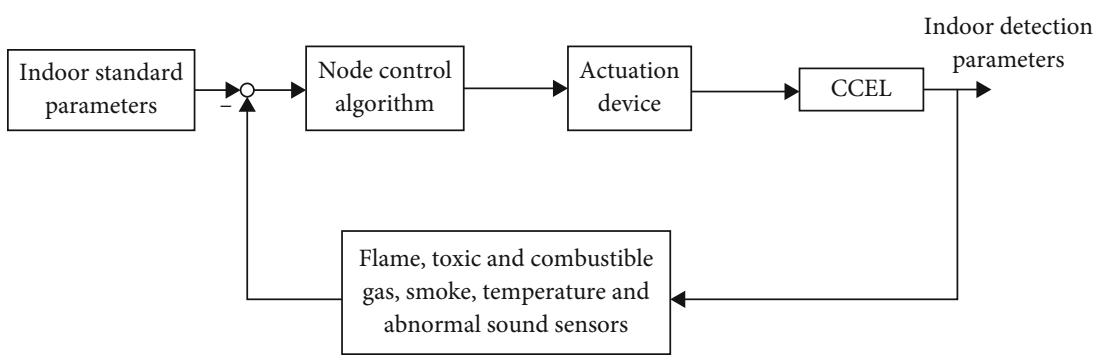

FIGURE 10: Structure diagram of measurement and control system in CCEL.

nodes (including the external sensors) and corresponding protective devices are fixed in $m$ buildings.

\subsection{Testing of Security Monitoring Node in CCEL. For exper-} imental verification, $m$ monitoring nodes are, respectively, installed in $m$ laboratories, the WSN node consists of several sensors, corresponding protective device, alarm system, and so on. The WSN monitoring node used in this experiment is shown in Figure 8.

The wireless monitoring node has internal and external sensors. The internal sensors are integrated on the monitoring node, such as temperature sensor and humidity sensor; they are applied to detect temperature and humidity inside this node. These external sensors are installed in suitable positions around the experimental devices. These sensors are connected to a wireless monitoring node via signal wires.

We put combustible gas, toxic gas, and smoke generators and the security monitoring node into one of the laboratories, then open the generators, as shown in Figure 9, which is the experimental wireless monitoring system diagram for CCEL. The system includes extinguishers, fume hood, WSN nodes, and gas generator. The WSN node is placed out of the laboratory to prevent the high temperature and fire damage. The signal line between sensors, extinguishers, and WSN node is coated with fireproof material. The fume hood can be used to release toxic and combustible gas when the gas concentration in the room exceeds the standard. The WSN node would alarm at the same time when the CCEL is in danger. Figure 10 illustrates the structure diagram of the measurement and control system in CCEL.

When we activate the monitoring system, the sensors start to monitor the laboratory data. And WSN node would transmit the data to and the handheld terminal and feed it back to the control system. If the detection parameters exceed the indoor standard parameters, the WSN node would control the actuation device to take a series of measures for the CCEL. For example, when there is a fire in the laboratory, the safety monitoring node turns on the fire extinguisher. The extinguisher sprays fire agent to the experiment device 


\begin{tabular}{|ll|}
\hline No.5/1002-1 & \\
$\mathrm{T} \geq 40{ }^{\circ} \mathrm{C}$ & $\mathrm{H} \leq 15 \%$ \\
$\mathrm{CG} \geq 10 \%$ & $\mathrm{TG}=0 \%$ \\
$\mathrm{TS}=0 \mathrm{~dB}$ & \\
SS: $\mathrm{Y}$ & \\
Alarm: & \\
\hline
\end{tabular}

(a)

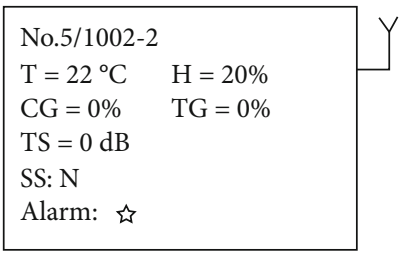

(b)

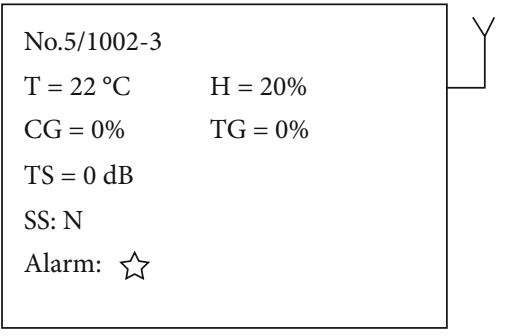

(c)

FIGURE 11: Testing experimental example 1 on the interface of the handheld terminal.

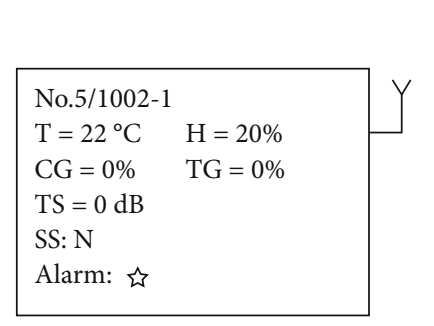

(a)

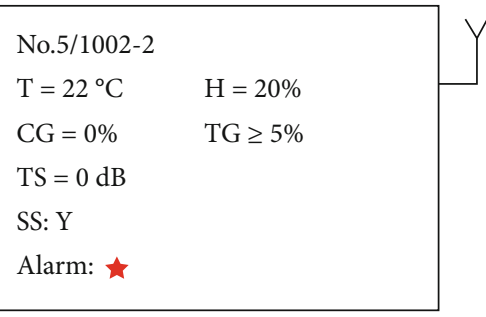

(b)

\begin{tabular}{|ll|}
\hline No. $5 / 1002-3$ & \\
$\mathrm{~T}=22{ }^{\circ} \mathrm{C}$ & $\mathrm{H}=20 \%$ \\
$\mathrm{CG}=0 \%$ & $\mathrm{TG}=0 \%$ \\
$\mathrm{TS}=0 \mathrm{~dB}$ & \\
SS: $\mathrm{N}$ & \\
Alarm: 公 & \\
\end{tabular}

(c)

FIGURE 12: Testing experimental example 2 on the interface of the handheld terminal.

in order to minimize the loss of laboratory equipment and to protect the safety of indoor staff.

5.3. Remote Testing Experiments Based on Handheld Terminal. This section introduces remote testing experiments based on handheld terminals. The remote test experiments in this paper were carried out in three laboratories, respectively. They are numbered 1002-1, 1002-2, and 10023 , and each laboratory covers an area of 50 square meters. When the indoor safety monitoring node detects smoke, flame, toxic gas leakage, and abnormal sound, the field information will be sent to the handheld terminal. The terminal will display the specific location of the alarm (i.e., which building? Which room? And the parameters indicated by the alarm (i.e., smoke, fire, gas leakage, and abnormal sound).
Alarm information will also be displayed on the handheld terminal, as shown in Figures 11-13.

5.3.1. Test Experiment Example 1. This experiment was conducted in the laboratories of 1002-1, 1002-2, and 1002-3, Teaching building No. 5, respectively. Let T stands for temperature, $\mathrm{H}$ for relative humidity, CG for combustible gas concentration, TG for toxic gas concentration, and TS for decibel value. SS stands for confirmation status (i.e. SS: N means normal; SS: Y means there is a dangerous accident). Alarm indicates the state of the Alarm (i.e., no sound on the Alarm indicates normal; a loud or flashing Alarm indicates a dangerous accident). When the concentration of combustible gas is measured to be greater than or equal to $10 \%$, and the indoor temperature rises and the relative humidity 


\begin{tabular}{|ll|}
\hline No.5/1002-1 & \\
$\mathrm{T}=22{ }^{\circ} \mathrm{C}$ & $\mathrm{H}=20 \%$ \\
$\mathrm{CG}=0 \%$ & $\mathrm{TG}=0 \%$ \\
$\mathrm{TS}=0 \mathrm{~dB}$ & \\
SS: $\mathrm{N}$ & \\
Alarm: 2 & \\
\hline
\end{tabular}

(a)

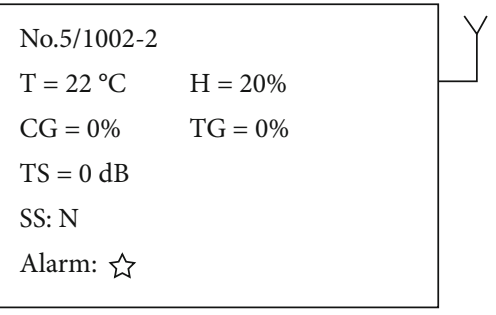

(b)

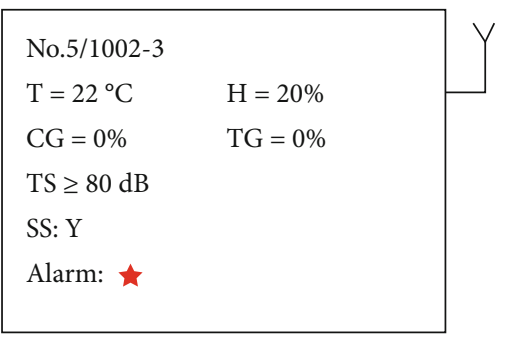

(c)

Figure 13: Testing experimental example 3 on the interface of the handheld terminal.

drops, the indoor combustion accident is considered to have occurred. The display information of the handheld terminal is shown in Figure 11, and its alarm information is shown in Figure 11(a).

(1)Automatic Protection Measures. Turn on the indoor camera, point the extinguisher at the ignition point, and spray dry powder extinguishing agent (or safety extinguishing agent) to quickly extinguish the ignition point. At the same time, send out an alarm signal to the indoor staff or nearby staff to evacuate the scene quickly.

5.3.2. Test Experiment Example 2. This experiment was conducted in the laboratories of 1002-1, 1002-2, and 1002-3, Teaching building No. 5, respectively. Let T stands for temperature, $\mathrm{H}$ for relative humidity, CG for combustible gas concentration, TG for toxic gas concentration, and TS for decibel value. SS stands for confirmation status (i.e., SS: N means normal; SS: Y means there is a dangerous accident). Alarm indicates the state of the Alarm (i.e., no sound on the Alarm indicates normal; a loud or flashing Alarm indicates a dangerous accident). When the toxic gas concentration is measured to be greater than or equal to $5 \%$, it is considered that a toxic gas leak has occurred in the room. The display information of the handheld terminal is shown in Figure 12, and its alarm information is shown in Figure 12(b).

(1)Automatic Protection Measures. Open the indoor fume hood or ventilator and at the same time send an alarm signal for indoor or nearby workers to evacuate the scene quickly.

5.3.3. Testing Experimental Example 3. This experiment was conducted in the laboratory of 1002-1, 1002-2, and 1002-3, Teaching building No. 5, respectively. Let T stands for temperature, $\mathrm{H}$ for relative humidity, CG for combustible gas concentration, TG for toxic gas concentration, and TS for decibel value. SS stands for confirmation status (i.e., SS: N means normal; SS: Y means there is a dangerous accident). Alarm indicates the state of the Alarm (i.e., no sound on the Alarm indicates normal; a loud or flashing Alarm indicates a dangerous accident). When the decibel value of the measured abnormal sound is greater than or equal to $80 \mathrm{~dB}$ (in this paper, it is assumed that the noise of more than $80 \mathrm{~dB}$ produced in the laboratory is an abnormal sound), it is considered that an explosion or an abnormal accident has occurred in the room. The display information of the handheld terminal is shown in Figure 13, and its alarm information is shown in Figure 13(c).

(1)Automatic Protection Measures. Turn on the indoor camera and activate the isolation device near the accident point. At the same time, send an alarm signal for the indoor staff or nearby staff to evacuate the scene quickly.

When doing experiments in the chemistry and chemical engineering laboratory, safety is of the first importance. Here, we give some safety measures that must be paid attention to during the experiment:

(1) When conducting fire experiment, it is necessary to have reliable fire extinguishing equipment and measures

(2) When conducting toxic gas experiments, the experiments can be carried out in a small space first, with reliable ventilation equipment and protective measures

(3) The isolation device and protective measures should be provided when conducting abnormal sound experiments

All in all, researchers should avoid major accidents during the experiment and must have protective equipment and safety measures. 


\section{Conclusion}

In view of the existing safety problems in chemical and chemical engineering laboratories, we propose a CCEL indoor safety wireless monitoring and automatic protection system. The testing results demonstrate that the main advantages of this system can be summarized as follows:

(1) The system proposed can achieve all-weather realtime monitoring of indoor safety information in CCEL, ensure that teaching experiments and scientific research experiments can be conducted continuously and safely, and significantly reduce the work intensity of laboratory managers. When the accident occurred, they were able to take effective measures to deal with it quickly and report relevant information to relevant departments in time, so as to reduce the damage caused by emergencies

(2) Because this system can in real-time monitor the safety status of CCEL, timely and accurately obtain the fire, toxic and harmful gas, abnormal sound, and other danger information of CCEL. Based on the acquisition of on-site monitoring data and image message, and it can remotely, wirelessly, and rapidly control the automatic protection device in CCEL. When an accident occurs, it will do its best to protect students and teachers working in the lab from harm and minimize damage to laboratory equipment

(3) The application of the system will solve a difficult problem in CCEL, and the system can be used for CCELs (such as toxic and hazardous materials warehouses and flammable and explosive places) of colleges, research institutes, vocational colleges, and colleges. Therefore, the system has a good application prospect

The limitations of the method proposed in this paper and the prospect of future research work are as follows:

(1) The specification standard of automatic fire extinguishing equipment applicable to the "gunpowder and explosives" chemistry and chemical engineering needs further study. A variety of automatic fire extinguishing methods are further studied, and the experimental results are analyzed and compared. The selection of fire-extinguishing materials (i.e., fireextinguishing agents) suitable for use in "gunpowder and explosives" chemistry and chemical engineering laboratories is required

(2) It is required to select a sensor that can quickly detect the toxic gas content so as to accurately find the leakage point of toxic gas. Once the toxic gas is detected, it is required to have a quick method to cut off the source of toxic gas. The effective methods and measures of automatic protection of experimental personnel and experimental equipment need to be further studied and improved
(3) When an explosion occurs in the laboratory, the system can automatically activate the protective device and quickly isolate the explosion site, so as to minimize casualties and property losses. These works still need to be further studied and improved

\section{Data Availability}

The effectiveness and availability of the proposed system are verified by the experimental results. Therefore, the experimental data supporting the results of this study (the experimental data type is number, i.e., including integer and decimal numbers) can be found in Section 5.3 of the manuscript or obtained from the corresponding author.

\section{Conflicts of Interest}

The authors declare that they have no conflicts of interest regarding the publication of this paper.

\section{Authors' Contributions}

Xiang-Guang Chen and Xue-Jiao Liu constructed the experimental system and designed the experimental scheme and carried out many experiments on the system in the laboratory. Qiao-Yan Sun and Xiang Han participated in several experiments of the system and studied the scheme of the automatic protection system. Yu-mei Sun and Mei-chun Wang reviewed references and materials (including recent years) related to the experiments of this system.

\section{Acknowledgments}

This scientific work is financially supported by the Yantai Double Hundred Plan Talent Project (YT201803) in 2018 and the Natural Science Foundation (No. ZR2016FM28) of Shandong Province in 2016.

\section{References}

[1] M.-Q. Bao, Y. Zhang, and S.-C. Zhang, "Analysis of college chemistry laboratory safety problems and exploration of safety management measures," Experimental Technology and Management, vol. 29, no. 1, pp. 188-191, 2012.

[2] Y.-q. Liu, H.-g. Bao, and S.-j. Song, "Research on University Laboratory Safety Management," Experiment Science \& Technology, vol. 10, no. 1, pp. 173-175, 2012.

[3] N.-w. Qiu, X.-c. An, J.-w. Jia, and Z. Kun, "Challenges and solutions confronted with for laboratory safety management in colleges and universities during new period," Experimental Technology and Management, vol. 29, no. 1, pp. 181-185, 2012.

[4] F. Jian, X. Wei, Q. Huidong, and L. Yanhui, "Exploration on strengthening the safety of chemistry and chemical engineering laboratory," Guangdong Chemical Engineering, vol. 43, no. 6, pp. 209-210, 2016.

[5] Q. Huidong, L. Guang, W. Wei, and L. Yanhui, "Comparison On safety management of chemistry and chemical engineering laboratories between universities of China and United States," Experimental Technology and Management, vol. 31, no. 4, pp. 2013-2015, 2014, 209. 
[6] Q. Qiu, Z. K. Luo, W. Z. Lu, and W. Fang, "Research of chemical laboratory safety management in domestic and abroad universities," Guangzhou Chemical Industry, vol. 38, no. 5, pp. 272-274, 2010.

[7] L. Zhi-Ming, C. Xiang-Guang, W. Lei, and W. Yanfu, "Fault diagnosis method for wireless security monitoring system in chemistry and chemical engineering laboratory," Transactions of Beijing Institute of Technology, vol. 35, no. 10, pp. 10621066, 2015, 1073.

[8] W. Ping and W. Zheng, "Design and implementation of open computer lab monitoring and management system," Computer and Modernization, vol. 11, pp. 125-128, 2007.

[9] W. Nie, J. Xu, and Y. Liu, "Design of automatic closed system about gas outburst monitor and its role of the reducing security cost," Proceedings of the 2nd International Conference Mine Hazards Prevention and Control, , pp. 538-543, Atlantis Press, Paris, France, 2010.

[10] W. Nie, Y. Liu, and J. Xu, "A gas monitoring and control system in a coal and gas outburst laboratory," Journal of Sensors, vol. 3, 11 pages, 2014.

[11] V. Ramya, B. Palaniappan, and V. Sumathi, "Gsm based embedded system for remote laboratory safety monitoring and alerting," International Journal of Distributed and Parallel Systems (IJDPS), vol. 3, no. 6, pp. 31-49, 2012.

[12] J. Linyiang, L. Hailong, and G. Zhenhua, "Embedded laboratory environment monitor system," in WASE International Conference on Information Engineering, pp. 197-201, Yaiyuan, China, 2009.

[13] L. Yang, L. Jiang, K. Yue, and P. Heming, "Design and implementation of the lab remote monitoring system based on embedded web technology," International Forum on Information Technology \& Applications, vol. 2, pp. 172-175, 2010.

[14] G. Zhao, Z. Wang, W. Li, and K. Wang, "An embedded laboratory security monitoring system," in Third International Conference on Measuring Technology and Mechatronics Automation, pp. 395-398, Shanghai China, 2011.

[15] G. Ying, C. Yan, and H. Fei, "Research of laboratory remote monitoring and early warning system based on wireless sensor network," Research \& Exploration in Laboratory, vol. 35, no. 3, pp. 105-109, 2016.

[16] L. Yu-yu, "Exploration of safety management for chemical laboratories in universities," Laboratory Science, vol. 18, no. 4, pp. 203-205, 2015.

[17] P. Yanling, "Construction study of the safety management system for chemical engineering laboratory," Modern Chemical Research, no. 2, pp. 11-12, 2017.

[18] F. E. N. G. Shou-chun, "Chemistry and chemical engineering laboratory safety management and safety education," Laboratory Science, vol. 17, no. 3, pp. 196-198, 2014.

[19] H. Xue, L. Yingling, Y. Guoqiang, and F. Guangzhu, "Exploration on the safety construction of chemistry and chemical engineering laboratory," Guangdong Chemical Industry, vol. 43, no. 13, p. 296, 288, 2016.

[20] L. Hai-Bin and J. Jie, "Safety management of chemical engineering laboratory in colleges and universities," Anhui Chemical Industry, vol. 49, no. 2, pp. 142-144, 2019.

[21] L. Yan, R. Shun-Lin, and H. Dong-Xian, "Exploration on safety management mechanism of chemical laboratories in universities," Guangzhou Chemical Industry, vol. 48, no. 5, pp. 198-199+211, 2020.
[22] L. Shiping, X. Aijuan, X. Bing et al., "Construction and practice of safety access system for laboratories of chemical engineering majors," Experimental Technology and Management, vol. 36, no. 11, pp. 11-14, 2019.

[23] G. Quanhui, T. Yanting, L. Juan, Z. Hui, and M. Xinqi, “Exploration on safety management of university chemical engineering laboratory under new normal situation," Experimental Technology and Management, vol. 34, no. 10, pp. 249-251, 2017.

[24] Z. Jìng, S. Qiao-Fang, J. Wu, and L.-X. Wang, "Construction and management of chemistry and chemical engineering laboratory in colleges and universities under the new situation," Chemical Industry Times, vol. 34, no. 2, pp. 39-41, 2020.

[25] W. Wu and C. Jian-Feng, "Exploration on the safety management system chemical laboratory in colleges and universities under the new situation," Chemical Enterprise Management, vol. 10, pp. 21-22, 2020.

[26] B.-J. Wan, “Analysis and preventive measures of safety hidden danger in chemical and chemical laboratories of local universities," Industrial \& Science Tribune, vol. 18, no. 11, pp. 234-235, 2019.

[27] Z. Bao-han, T. Qiang, W. Wei et al., "Comparison of safety management between Chinese and Norwegian chemical laboratories," Experimental Technology and Management, vol. 27, no. 4, pp. 49-50, 2019.

[28] Z. Dong, M. Kai-Yuan, J. Nan, L. Ting-Ting, and L. Sen-Lín, "A brief analysis on the construction of university laboratory safety management system based on information platform," China Inspection Body \& Laboratory, no. 4, pp. 49-50, 2019.

[29] Y.-m. Sun, X.-j. Liu, X.-g. Chen, Q.-y. Sun, and Z. Jun, "Research and application of a fault self-diagnosis method for roots flowmeter based on WSN node," Wireless Personal Communications, vol. 59, no. 3, pp. 2315-2330, 2017.

[30] Y.-M. Sun, X.-J. Liu, Q.-Y. Sun, and X.-G. Chen, "Research and application of wireless security monitoring system in chemistry and chemical engineering laboratory," Wireless Personal Communications, vol. 59, no. 3, pp. 2331-2344, 2017.

[31] L. Zhi-Min and C. Xiang-guang, "Research and application of WSN node communication energy consumption model based on asynchronous MAC protocol," Transactions of Beijing Institute of Technology, vol. 35, no. 2, pp. 171-175, 2015.

[32] L. Zhimin and X. Cheng, "Research on fault diagnosis of wireless sensor node on module level," Chinese Journal of Scientific Instrument, vol. 34, no. 12, pp. 2763-2769, 2013.

[33] X. Ying-Xin, C. Xiang-Guang, and Z. Jun, “A double weighted LS-SVM model for data estimation in wireless sensor networks," Journal of Beijing Institute of Technology, vol. 21, no. 1, pp. 134-139, 2012.

[34] Z. Jun, C. Xiang-Guang, L. Chun-Tao, Y. Xiang-Ming, and Y. Bin, "Study on application of distributed multi-channel MAC protocols in WSN," Acta Armamentarii, vol. 33, no. 6, pp. 695-701, 2012. 